\title{
ROLE OF CLINICAL PHARMACIST IN CLINICAL AUDIT AT DEPARTMENT OF GENERAL MEDICINE
}

\author{
P. Tasleem*1, M.Reshma ${ }^{1}$, M. Sai Kiran ${ }^{1}$, Dr. L.Reddenna ${ }^{2}$, Dr. V.Sreedhar ${ }^{2}$ \\ ${ }^{I}$ Department of Pharmacy Practice, Parma D Intern, Balaji College of Pharmacy, Andhra Pradesh, \\ Anantapuramu-515001 \\ ${ }^{2}$ Department of Pharmacy Practice, Associate Professor, Ph D, Balaji College of Pharmacy, Andhra \\ Pradesh, Anantapuramu-515001
}

Article Info: Received 20 December 2020; Accepted 28 January. 2021

DOI: https://doi.org/10.32553/jbpr.v10i1.826

Corresponding author: P. Tasleem

Conflict of interest statement: No conflict of interest

\begin{abstract}
:
Background of the study: Inaccuracy in writing and poor legibility of handwriting or incomplete writing of a prescription can lead to misinterpretation, thus leading to errors in dispensing and administration. Both individual and system related factors are responsible for prescribing errors. Recognition is the essential step in building safer systems and preventing errors. These errors can be detected by systematic analysis of prescriptions through a prescription audit. Hence, this project was proposed to improve the prescription practice by recommending the prescribing and therapeutic interventions in a selected setting of Anantapur.

Research Methodology: The study was approved by the ethical committee of the hospital. Before starting the study, the researcher obtained formal permission from Government General Hospital, Anantapuramu. The data collection period was for 6 months. A prospective observational design was adopted for this study. The prescriptions were selected on the basis of inclusion criteria. Descriptive statistics like frequency and percentage were calculated to describe the data.

Results and discussion: The overall observation made from this study was that clinical pharmacist could identify some drug related problems, provides clinical pharmacy services and contribute to better patient care being a part of the healthcare team.

Conclusion: There is enormous scope for the clinical pharmacist to play an important role in health care in various aspects like monitoring ADRs, provision of drug information, identifying DRPs and patient counseling which will bring better patient outcome.
\end{abstract}

Key words: Adverse drug reactions, Clinical audit, Clinical Pharmacist, prescription audit

\section{Introduction}

Clinical audit could be a quality improvement method that seeks to improve patient care and outcomes through systematic review of care against unambiguous criteria and also the implementation of amendment. Aspects of the structure, processes, and outcomes of care are elected and consistently evaluated against unambiguous criteria. Where indicated, changes are implemented at an individual, team, or service level and further monitoring is used to confirm improvement in healthcare delivery. Clinical audit can be described as a cycle or a spiral. Within the cycle there are stages that follow a systematic process of establishing best practice, measuring care against criteria, taking action to improve care, and monitoring to sustain improvement. The spiral suggests that as the process continues, each cycle aspires to a higher level of quality. Clinical audit can be undertaken by individual healthcare staff, or groups of professionals in single or multidisciplinary teams. For clinical governance to fulfill its promise, new skills are required, including improved understanding of clinical audit and of the 
need for an organizational environment that supports effective clinical audit.

As a quality improvement tool, audit can demonstrate that real efforts are being made by dedicated, hard-pressed staff to deliver high-quality professional care to all their patients.

Clinical pharmacy activities include medication history reviews on admission, medication management reviews during in-patient episodes, patient medication counseling, clinical reviews, therapeutic drug monitoring, and selection of drug therapy, adverse drug reaction monitoring and provision of drug information. ${ }^{1}$

Pharmacy practice has evolved dramatically over the last four decades. In many settings, the pharmacist's role has shifted from a primary focus on medication dispensing to a focus on providing patient care. Historically, the practice of pharmacy has been that of interpreting, evaluating, and implementing medical orders and dispensing medications. However, the practice of pharmacy has expanded to include determining optimal evidence-based medication management, monitoring for adverse drug events, educating patients and caregivers on medication use, and collaborating with physicians and other health professionals in the management of acute and chronic diseases.

The literature is replete with publications that describe the expanding role of pharmacists in a variety of practice settings. The pharmacist's role has been documented in the hospital setting illustrating cost control and reduction, antimicrobial policy adherence, optimization of antimicrobial use, prevention of adverse drug events, pain and anticoagulation management, and the detection and control of hypertension. ${ }^{2}$

Pharmacists have an important role in decreasing medication misuse through collaborative drug therapy. This role is not new for pharmacists. Studies demonstrate that pharmacist recommendations can prevent medication errors and minimize adverse effects. Other studies have shown that pharmacist recommendations can decrease the cost of therapy and improve outcomes. ${ }^{3}$

Pharmacists are well positioned to conduct research to:

1. Identify problems encountered in daily practice that contribute to the gap between recommended care and actual care and,
2. Demonstrate whether treatments with proven efficacy are truly effective and sustainable when used in practice. ${ }^{4}$

\section{Research Methodolgy}

Research Approach: Qualitative approach was used for this study. "Qualitative approach is concerned with understanding the behavior of healthcare professionals to explore types of practices and beliefs in order to improve them."

Research Design: Prospective observational design was adopted for the study.

Setting of the Study: The study was conducted at Government General Hospital, Ananthapuramu.

Study Population: In this study, the target population was the prescriptions/medication orders of the patients from Government General Hospital, Ananthapuramu.

Sample: Samples of the study were the prescriptions/medication orders of the patients from Government General Hospital, Ananthapuramu, who fulfilled the inclusion criteria with total comprised of 326 .

Sampling Technique: "Simple random sampling is a sampling technique where every item in the population has an even chance and likelihood of being selected in the sample." Thus in this research, simple random sampling technique was used to select the samples.

\section{Criteria for Sample Selection:}

\section{Inclusion Criteria}

- Prescriptions containing at least two medications in a selected hospital

- Prescriptions of patients who were more than 18 years of age.

\section{Exclusion Criteria}

- Prescriptions of patients with multiple comorbidities

- Prescriptions of pregnant women and lactating mothers

Data Collection Procedure: The main study was conducted after the approval of the ethical committee of the hospital. Before starting the study, the researcher obtained formal permission from Government General Hospital, Ananthapuramu. The data collection period was for 5 months.A prospective observational design was adopted for 
this study. The prescriptions were selected on the basis of inclusion criteria. The techniques used for data collection were interview and record analysis. The details of all the prescriptions were analyzed on the following parameters:

- Prescription format and its completeness

- Legibility of prescriptions

- WHO core prescribing indicators

- Drug related problems

Data Analysis:

After the data collection, data was organized, tabulated, summarized \& analyzed using descriptive statistics and inferential statistics according to the objectives of the study. Descriptive statistics like frequency, percentage, mean, standard deviation were calculated to describe the data. Inferential statistic like student t- test was calculated to infer the data.

\section{Results:}

\section{Clinical Audit}

A total of 326 cases were screened for clinical audit

\section{Gender Wise Distribution of Samples}

Among 326 patients involved in the study the males were 180(55\%) and females were 146(36\%)

\section{Age Wise Distribution of Samples}

All the patients were categorized into 7 age groups, showed that number of patients between the age group of 41-50 years were $90(28 \%)$ and number of patients between the age group of $>81$ years were $10(4 \%)$

\section{Prescription Audit}

\section{Correlation of Basic Information With Prescriptions}

A total of 326 medication orders were screened for prescription auditing, out of which 75 medication orders had different types of problems like $8(11 \%)$ prescriptions had found without names, 10(13\%) without age, 6(8\%) without gender ,20(27\%) complete address $2(3 \%)$ without diagnosis, 4(5\%) without department,25(33\%) without signature.

\section{Prescription Errors:}

Out of 326 medication orders screened for prescribing standards, the prescribing errors (100) in which dose 30(30\%), dosage form 15(15\%), frequency $30(30 \%)$ duration of treatment $25(25 \%)$

\section{Type of Drugs:}

A total of 326 medication orders were screened, out of which 295(90\%) were generic names and $31(10 \%)$ were brand names.

\section{Legibility of Prescriptions:}

Out of 326 medication orders screened for prescription auditing, in which 115 (35\%) prescriptions were legible and 211 (65\%) were illegible.

\section{Correlation of Drugs With Prescriptions}

Out of 326 medication orders screened, 4-5 drugs were 197 (60\%), 6-7 drugs were $1160(34 \%)$ and $>7$ drugs $19(6 \%)$

\section{Correlation of Antibiotics With Prescriptions}

A total of 326 medication orders screened, only one antibiotic was prescribed in 260 (80\%) prescriptions, two antibiotics in 52 (16\%), three antibiotics in $14(2 \%)$ as shown in the table.

\section{Fixed Dose Combinations}

Out of 326 medication orders screened, 164 (45.3\%) prescriptions contain fixed dose combinations.

\section{Vitamins}

Out of 326 prescriptions screened, 203 (56\%) prescriptions contain vitamin supplements.

\section{Who Prescribing Indicators}

a) Percentage of drugs prescribed by generic name $=$ (Number of drugs prescribed by generic name/Total number of drugs prescribed) $\times 100$

\section{$1073 \div 1182 \times 100=90.1 \%$}

b) Percentage of the prescription with an antibiotic prescribed $=($ Number of the prescription with antibiotics/Total number of prescription $) \times 100$

$$
326 \div 326 \times 100=100 \%
$$

According to WHO Prescribing indicators, percentage of drugs by generic name is $90 \%$, and percentage of prescriptions with an antibiotic prescribed is 100 .

\section{Therapeutic Audit}

A total of $\mathbf{3 2 6}$ medication charts were reviewed from June 2019 to December 2019, according to the inclusion criteria. During the study period, Clinical Pharmacist carried out various activities like 
identifying drug related problems, patient counseling and answering drug information queries.

Table 1: Clinical pharmacy activities

\begin{tabular}{ll}
\hline Adverse drug reactions & 43 \\
\hline Medication Errors & 08 \\
\hline Drug-Drug Interactions & 10 \\
\hline Drug-Lab Interactions & 07 \\
\hline Drug-Disease Interactions & 04 \\
\hline Patient Counseling & 21 \\
\hline
\end{tabular}

\section{Gender Wise Distribution of Adrs:}

From the 326 charts reviewed, 43 ADRs were found, out of which $24(55.82 \%)$ were male and $19(44.18 \%)$ were female.

\section{Distribution of Adrs: Age Wise}

Majority of ADRs 11(25.59\%) were found in the age group of 41-50 years, and 4(9.31) in 11-20 age group, 5(11.62) in 21-30 age group, 6(13.95) in 3140 age group,7(16.27) in 51-60 age group,4(9.31) in 61-70 age group,6(13.95) in 71-80 age

\section{Therapeutic Class Wise Distribution of Adrs}

Maximum no. of ADRs were caused due to NSAIDs $10(23.25 \%)$ followed by antidaibetic $6(13.95 \%)$, antibiotics $5(11.62 \%)$ and steroids $5(11.62 \%)$ and also along with that in anti tubercular, anti hypertensive is $4(9.320$, sedatives, ant malarial, anti viral ,anti epileptic is 1(2.32)

\section{Severity of Adrs}

From the 43 cases severity of adrs were found to mild 8(18.62) , moderate 23(53.48), severe 12(27.90)

\section{Causality Assessment of Adrs}

In the probability scale the number of ADRs were found to be highly probable 2(4.66), probable 23(53.48), possible 18(41.26) by using of Naranjo's probability assessment scale

Table 2: Details of drugs causing ADRs

\begin{tabular}{lll}
\hline SI No. & Drugs & Type of ADRs \\
\hline 1. & Ceftriaxone & Skin rashes \\
\hline 2. & Ranitidine & Skin rashes \\
\hline 3. & Ciprofloxacin & Skin rashes \\
\hline 4. & Aspirin & Skin rashes/itching \\
\hline 5. & Theophylline & Fever with chills \\
\hline 6. & Metformin & Hypoglycemia \\
\hline 7. & Glipizide & Hypoglycemia \\
\hline 8. & Diclofenac & Gastric ulcer \\
\hline
\end{tabular}

\section{Drug Related Problems (Drps)}

From the 326 medication charts reviewed, 29 DRPs were found. Out of the 29 DRPs, 10 were due to drug-drug interactions, followed by medication errors 8 ( 6 belonged to drugs without indication, 2 were pertaining to same class of drugs), 7 were due to drug- lab interactions, 4 due to drug-disease interactions.

Table 3: Drug-Drug interactions leading to drps

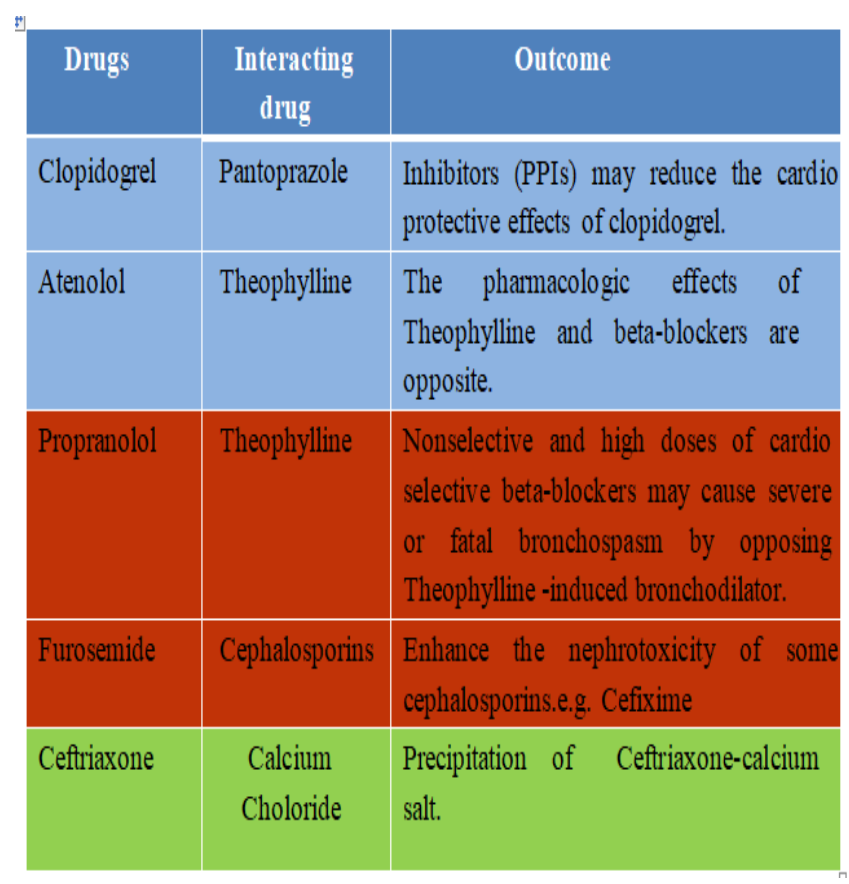

\section{CLINICAL SIGNIFICANCE OF DRUG-DRUG INTERACTIONS}

The clinical significance of the documented drugdrug interactions was analyzed and is shown in the figure -1

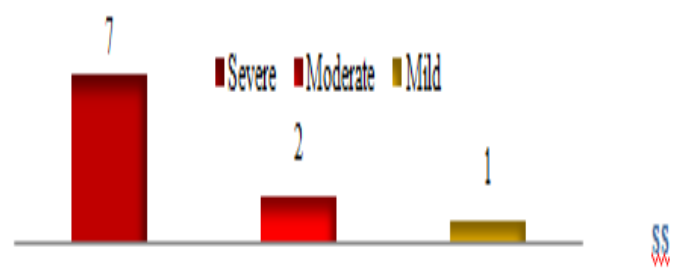

Figure 1: Significance of Drug-drug interactions

\section{Patient Counseling:}

Twenty one in-patients were counseled during the study for various diseases and their medications, which includes

- Hypertension 
- Diabetes

- Asthma

\section{Queries Answered}

During the study period, a total of 18 queries were answered raised by physicians, nurses and patients/patient caretakers. The categorical distribution of drug information queries is shown in table.

Table 4: Categories of drug information queries

\begin{tabular}{ccc}
\hline $\mathbf{1 .}$ & Drug Profile & 8 \\
\hline $\mathbf{2 .}$ & Indication/Dose & 2 \\
\hline $\mathbf{3 .}$ & Adverse Effects & 3 \\
\hline $\mathbf{4 .}$ & Management & 1 \\
\hline $\mathbf{5 .}$ & Drug Interaction & 2 \\
\hline $\mathbf{6 .}$ & Pregnancy/Lactation & 1 \\
\hline $\mathbf{7 .}$ & Poisoning & 1 \\
\hline
\end{tabular}

\section{Acceptance of Interventions}

During the study period, a total of 29 interventions were recommended, out of which 16 interventions were accepted.

\section{Discussion:}

Clinical pharmacy services help in achieving better patient outcome by contributing towards optimization of drug therapy.

The pharmacist audited the prescriptions from outpatient department as a part of prescription audit. The pharmacist participated in ward rounds and reviewed the medication charts as a part of therapeutic audit, for identifying adverse drug reactions, drug interactions and medication errors. The pharmacist communicated his observations to the Head of the unit and documented the outcome. The pharmacist also answered queries raised by the medical team during the ward rounds and also carried out 'patient counseling'.

\section{Prescription audit:}

Legibility problem encounters the foremost medication error in wards and sometimes proven to be fatal in emergency cases. We encountered an instance when a nurse administered tablet flupenthixol instead of tablet

During the study period, a total of 326 medication charts were reviewed, out of which 43 adverse drug reactions were documented. The incidence of ADRs was high, 11(25.59\%) in the age group of 41 to 50 years. This may be because patients of this age group were taking multiple medications for several co- morbid conditions. By using Hartwig's Severity Assessment Scale, it was found that 23(53.48\%) ADRs were of moderate severity. This finding correlates with the study of KJ Patel et al., where most of the ADRs were found to be of moderate severity $(74.71 \%){ }^{5}$ the most severe ADR was found to be gastric ulcer caused by Diclofenac.

The class of drugs causing maximum number of ADRs [10(23.25\%)] was NSAIDs followed by antidaibetic [6(13.95\%)], antibiotics [5(11.62\%)] and steroids [5(11.62\%)]. These findings correlate with the study conducted by Saha L, where most of the adverse effects $(22 \%)$ were attributed to the use of NSAIDs. ${ }^{6}$

According to the Naranjo's Probability Assessment Scale there were [2(4.66\%)] Highly Probable, [23(53.48\%)] Probable and [18(41.86\%)] Possible ADRs. This finding is in contrast to the findings of E.C.Davies where most number of ADRs fall under possible category. ${ }^{7}$

During the medication chart review, the pharmacist identified 8 medication errors, out of which, 6 were due to prescribing drugs without indication, Two medication errors were identified wherein the same class of drugs viz omeprazole and ranitidine were prescribed. These findings correlate with study of Bhupathy Alagiriswami where the medication errors were attributed to use of drugs without indication. $^{8}$

A total of 18 queries were asked by the clinicians out of which majority of queries (8) were pertaining to drug profile. All primary, secondary and tertiary resources were utilized for answering the queries.

Being a part of the medical team, the clinical pharmacist was directly able to provide counseling to 21 in-patients at their bedside. Patients/patient care takers (in cases where patients were not responding) were counseled not only about the medication, but also about better management of the disease.

The acceptance rate of intervening clinical pharmacist recommendations was found to be $55.17 \%$. $44.82 \%$ of the interventions were not accepted; some of the reasons may be due to nonavailability of other alternatives or the benefit ratio being higher than risk ratio. This finding is in contrast with the study of Ganachari M S where the acceptance rate was $78.37 \% .{ }^{9}$ another reason may 
be that prescribing decisions are often governed by clinical experience of physicians. This indicates that a clinical pharmacist can contribute to better patient care if made a member of the health-care team.

The overall observation made from this study was that clinical pharmacist could identify some drug related problems, provides clinical pharmacy services and contribute to better patient care being a part of the healthcare team.

\section{Conclusion:}

The clinical pharmacist being a part of the health care team in the medicine ward could contribute to patient in the following ways.

- In detection and monitoring of Adverse Drug Reactions: 43 ADRs were identified and analyzed in detail regarding their causality and severity.

- In providing unbiased drug information which can promote the safe, effective and rational use of drugs: Information was provided to clinicians for the drug information queries (18) raised by them most of them being related to patient management.

- In identifying Drug Related Problems: 18 medication errors, 10 drug-drug interactions, 4 drug-disease interactions and 7 drug-lab interactions were observed from the medication chart review which were informed to the Unit Head.

- In providing Patient counseling: Medication as well disease counseling helped the patients in better understanding of their disease and the drugs.

Out of the number of interventions suggested by the clinical pharmacist, $55.17 \%$ were accepted by the clinicians and implemented.

Thus, from this study it was concluded that there is enormous scope for the clinical pharmacist to play an important role in health care in various aspects like monitoring ADRs, provision of drug information, identifying DRPs and patient counseling. Thus, pharmacist can positively intervene in drug therapy which will bring about better patient outcome.

\section{Reference:}

1. Dooley MJ, Allen KM, Doecke CJ, Galbarth $\mathrm{KJ}$, Taylor GR, Bright $\mathrm{J}$ et al. A prospective multicentre study of pharmacist initiated changes to drug therapy and patient management in acute care government funded hospitals. $\mathrm{Br}$ J Clin Pharmacol 2003 Oct;57(4):513-521.

2. Pamela L, George D. Documentation of pharmacists' interventions in an emergency department and associated cost avoidance, Am J Health-Syst Pharm 2007 Jan 1; 64:63-64.

3. Audrey JL, Maureen SB, Katherine KK, Joy LM, and Nancy EK. Clinical and economic outcomes of pharmacist recommendations in a veteran's affairs medical center, Am J HealthSyst Pharm 2002 Nov 1; 59:2070.

4. Lourdes GP. Intervention design, Implementation and Evaluation, Am J HealthSyst Pharm 2008 Oct 1; 65:1854-1859.

5. Patel KJ, Kedia M S, Bajpai D, Mehta S S, Kshirsagar N A, Gogtay N J. Evaluation of the prevalence and economic burden of adverse drug reactions presenting to the medical emergency department of a tertiary referral centre: a prospective study. BMC Clinical Pharmacology 2007;7(8):1-5.

6. Saha L, Pandhi P, Malhotra S, Sharma N. Adverse drug event related medical emergency department visits and hospital admissions: a prospective study from a north Indian referral hospital. Journal of Clinl Dia Rech 2008 Feb; (2):600-604.

7. Davies EC, Tejal K, David WB. Identifying drug safety issues: from research to practice. International journal for quality in health care 2000;12(1):69-76.

8. Alagiriswami B, Ramesh $\mathrm{M}$, Parthasarathi G, Basavana gowdappa H. A study of clinical pharmacist initiated changes in drug therapy in a teaching hospital ijopp 2009 Jan;1(2):36-45.

9. Ganachari MS, Mahendra Kumar BJ, Shashikala WC, Fibin M. Assessment of drug therapy interventions by clinical pharmacist in a tertiary care hospital. ijopp $2010 \mathrm{Jul} ; 3$ (3):22-28 